\title{
Characterization of melanin extracted from apricot (Armeniaca sibirica) and its effect on hydrazine-induced rat hepatic injury
}

\author{
Hong-jiao Li ${ }^{\mathrm{a}, \mathrm{b}}$, Ju-xiu Li ${ }^{\mathrm{c}}$, Zhong Zhao ${ }^{\mathrm{a}, *}$ \\ ${ }^{a}$ College of Forestry, Northwest A\&F University, and \\ Key Laboratory of Environment and Ecology in Western China, Ministry of Education, Yangling, \\ Shaanxi, 712100, China \\ b College of Forestry, and Forestry Post-Doctoral Research Centre, Agricultural University of Hebei, \\ Baoding, Hebei, 071001, China \\ c College of Food Technology and Engineering, Northwest A\&F University, Yangling, Shaanxi, 712100, \\ China
}

*Corresponding author, e-mail: zhaozh@nwsuaf.edu.cn

Received 14 Jul 2015

Accepted 15 Feb 2016

\begin{abstract}
Melanin is a complex, highly coloured natural antioxidant with potent biological activities and diverse applications. A potential source of melanin is the Siberian apricot (Armeniaca sibirica) whose kernel skin is currently a waste by-product. To explore its potential, melanin from apricot kernel skin was extracted. The molecular mass was determined by gel chromatography and its structure was examined by elemental analysis, UV-Vis, infrared, and solid-state nuclear magnetic resonance $\left({ }^{13} \mathrm{C}-\mathrm{NMR}\right)$ spectroscopy. In addition, its protective effect against hydrazineinduced hepatic injury in rats was evaluated. The results showed that melanin has $<5 \%$ sulphur (S) content, indicating that it consists mostly of eumelanin. Its UV-Vis, infrared and ${ }^{13} \mathrm{C}$-NMR spectra were very similar to those of synthetic melanin, although it had relatively high carboxylate, aliphatic, and phenol-skeleton content. Two distinct fractions $(2.4 \pm 0.12$ and $0 . \pm 560.1 \mathrm{kDa})$ were detected. Injections of hydrazine at $65 \mathrm{mg} / \mathrm{kg}$ body-weight (b.wt.) doses resulted in significant $(p<0.01)$ increases in lipid peroxidation (malondialdehyde contents) in liver homogenates and serum alanine aminotransferase and aspartate aminotransferase activities $(64 \%, 69 \%$, and $98 \%$ increase relative to the controls, respectively). It also induced significant ( $p<0.05$ or 0.01 ) decreases in liver homogenate catalase, glutathione peroxidase, and superoxide dismutase activities and reduced glutathione content (48\%, 28\%, 7\%, and 98\% reduction, respectively). Administering melanin prior to hydrazine, at $10 \mathrm{mg} / \mathrm{kg}$ b.wt., strongly inhibited these changes and ameliorated histopathological hepatic injury. The results indicated that melanin alleviated hydrazine-induced liver injury by relieving oxidative stress, which may provide a novel protective strategy for protection against liver diseases.
\end{abstract}

KEYWORDS: apricot kernel, physicochemical characterization, protection

\section{INTRODUCTION}

Melanin is a complex, high molecular weight substance that is composed of phenolic or indolic compounds and is widespread in the biosphere. As a result of physicochemical properties that include heavy metal chelation, radical scavenging and strong UV absorption ${ }^{1,2}$, it has important biological activities, inter alia hepatoprotection, and plays key roles in stress resistance ${ }^{3,4}$. Melanin is also used for diverse purposes in the pharmaceutical ${ }^{3,5}$, cosmetic $^{6,7}$, food ${ }^{8}$, electronics 9 , and other industries ${ }^{10,11}$, as it has strong antioxidant activities, anti-radiation functions, anti-virus functions, hep- atoprotective effects, and photoconductivity. It can be obtained by chemical synthesis from tyrosine or its derivatives ${ }^{12,13}$, or extracted from either animals $^{14,15}$ or plants ${ }^{16,17}$. Some microorganisms also produce melanins ${ }^{18}$, with physical and chemical properties that include high molecular weight, insolubility in water ${ }^{19}$, aqueous acids and common organic solvents, decolourization by oxidizing agents $\left(\mathrm{KMnO}_{4}, \mathrm{NaOCl}\right.$, and $\left.\mathrm{H}_{2} \mathrm{O}_{2}\right)$, metal ion chelating ability, and strong UV-Vis absorption. Melanins from plants have higher stability and wider applications, but there is little information on their structure. However, the composition of all melanins is known to depend both on their monomeric units and con- 

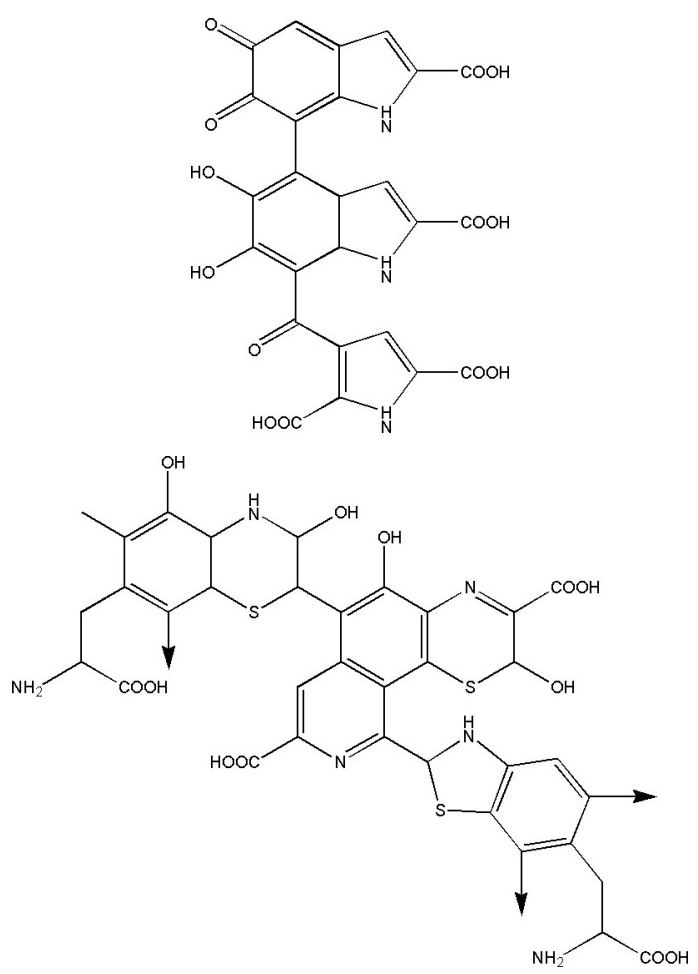

Fig. 1 Structural models of (top) eumelanin and (bottom) pheomelanin.

ditions during polymerization. There are three general forms; black-brown eumelanin, pheomelanin (ranging from red to yellow brown), and black allomelanin. The structures of eumelanin and pheomelanin are still unclear, but basic structural models suggested by Nicolaus and Swan ${ }^{20}$ have been widely adopted (Fig. 1).

Natural colourants and antioxidants, such as melanins, are increasingly popular among consumers because they often regard synthetic alternatives as undesirable or harmful ${ }^{7,21}$. Thus there is increasing interest in these substances and their sources $^{22,23}$. Siberian apricot (Armeniaca sibirica), a member of the Prunoideae subfamily of Rosaceae, is a natural hybrid that is widely distributed in northern China, where it plays important roles in afforestation, conservation of soil and water, and commercial production of edible fruit pulp and oil, which has various applications ${ }^{24}$. Apricot kernel skin (testa), accounting for about $5 \%$ of apricot mass, is discarded as waste. However, it has high melanin contents, and thus is a potentially valuable source of melanin. There is however little information on the structure of this melanin and methods for extracting it.
The liver, the largest organ in the human body, plays a vital role in clearing diverse toxins and xenobiotics ${ }^{25-27}$. Partly because several kinds of free radicals are produced in the liver during their elimination, various deleterious agents can induce inflammation, fibrosis, necrosis, and eventually cirrhosis and functional deterioration of the organ ${ }^{28}$. Acute and chronic liver diseases are of global concerns, and medical treatments often involve difficult protocols and have limited efficiency ${ }^{29}$. Hence, there is considerable interest in complementary or alternative medicines for their treatment ${ }^{26,30}$, particularly drugs from natural products, which may have lower toxicity than synthetic drugs ${ }^{5}$.

One severely deleterious agent is hydrazine. It is a mutagenic compound that is responsible for the hepatotoxicity of the anti-tubercular medicine isoniazid and increases risks of systemic diseases and cancer ${ }^{5,31}$. Thus in the present study, melanin from apricot kernels (MAKS) was isolated, and its physicochemical properties were examined. In addition, its hepatoprotective capacity was evaluated by testing the ability to protect rat liver from injury induced by hydrazine, using several wellcharacterized enzymes as biomarkers.

\section{MATERIALS AND METHODS}

\section{Materials}

Kernels were obtained from fruits collected from wild apricot trees in Pingliang, Gansu Province, China. After manual peeling, the apricot kernel skin was air dried before using. Synthetic melanin, hydrazine, Superdex G-75, bovine serum albumin, carbonic anhydrase, cytochrome $\mathrm{C}$, and vitamin $B_{12}$ were purchased from Sigma Chemical Co (St. Louis, MO). Kits for assaying alanine aminotransferase (ALT), aspartate aminotransferase (AST), superoxide dismutase (SOD), catalase (CAT), glutathione peroxidase (GSH-Px) activities, as well as reduced glutathione(GSH) contents and lipid peroxidation (malondialdehyde, MDA) were purchased from Nanjing Jiancheng Bioengineering Institute, Nanjing, China. All other reagents used were of analytical grade.

\section{Melanin isolation and purification}

Melanin was isolated and purified as follows. Samples of $20 \mathrm{~g}$ apricot kernel skin were broken into pieces and boiled in water at 1:15 solid:liquid volume ratio for $5 \mathrm{~min}$ then the resulting mixture was filtered using a filter cloth. MAKS was extracted from the solid residue in $0.4 \mathrm{M} \mathrm{NaOH}$ solution 
at $1: 20$ solid:liquid volume ratio for $6 \mathrm{~h}$ at $80^{\circ} \mathrm{C}$ in a water bath. The mixture was re-filtered to remove solid matter, and centrifuged at $2100 \mathrm{~g}$ for 10 min. The supernatant was acidified to $\mathrm{pH} 2.0$ by adding $2 \mathrm{M} \mathrm{HCl}$, incubated at room temperature for $2 \mathrm{~h}$, and then centrifuged again at $2100 \mathrm{~g}$ for $15 \mathrm{~min}$. The resulting dark, MAKS-containing pellet was suspended in $6 \mathrm{M} \mathrm{HCl}$ and incubated for $2 \mathrm{~h}$ in a boiling water bath, then centrifuged at $2100 \mathrm{~g}$ for $10 \mathrm{~min}$ to remove the liquid residue. The precipitate was washed with ethanol until the supernatant was colourless. The residue was dissolved by incubation in $0.4 \mathrm{M} \mathrm{NaOH}$ for $2 \mathrm{~h}$ in an $80^{\circ} \mathrm{C}$ water bath. The steps from acidification to the final centrifugation were repeated until this step yielded a supernatant with a $\mathrm{pH}$ of about 7.0. The recovered material was vacuum-dried to obtain pure melanin.

\section{Elemental analysis}

The elemental amounts of carbon(C), hydrogen (H) and nitrogen $(\mathrm{N})$ in MAKS were determined using a Vario EL elemental analyser (Elementar, Germany), following standard protocols ${ }^{1,21}$. The amount of $S$ in the MAKS was determined by the oxygenvolumetric flask method.

\section{UV-Vis absorption spectra of the melanins}

To determine the UV-Vis absorption spectra of MAKS and of the synthetic melanin, one sample of each was dissolved in ammonia at $0.2 \%$, with ultrasonic assistance, and then the ammonia was removed using a rotary evaporator ${ }^{21,32}$. The resulting solutions were scanned in a U-3310 UV-Vis spectrophotometer (Hitachi, Japan) to obtain absorption spectra at wavelengths ranging from 190-700 nm.

\section{Infrared spectra of the melanins}

Infrared spectra of both melanins were recorded, from 4000 to $400 \mathrm{~cm}^{-1}$, using an FT-IR330 infrared spectrophotometer (Thermo Electron Corporation, U.S.A) with $\mathrm{KBr}$ powder ${ }^{21,32}$.

\section{Evaluation of molecular mass}

The molecular mass of the MAKS was measured following Sava ${ }^{33}$. MAKS was dissolved in ammonia at $0.2 \%$, with ultrasonic assistance, then ammonia in the sample was removed using a rotary evaporator $^{21,32}$. After filtering the resulting solution through a $0.45 \mu \mathrm{m}$ syringe filter, MAKS was separated on a Superdex G-75 column $(1.6 \times 40 \mathrm{~cm})$ by gel chromatography in $50 \mathrm{mM}$ phosphate buffer
(pH 7.5) at a flow rate of $0.5 \mathrm{ml} / \mathrm{min}$. The eluate was monitored at $280 \mathrm{~nm}$, and the molecular mass of fractions with peaks at this wavelength was estimated using bovine serum albumin (66 kDa), carbonic anhydrase $(29 \mathrm{kDa})$, cytochrome $\mathrm{C}(12.4 \mathrm{kDa})$, aprotinin $(6.5 \mathrm{kDa})$, and vitamin $\mathrm{B}_{12}$ (1.36 kDa) as calibration standards.

\section{${ }^{13} \mathrm{C}-\mathrm{NMR}$ analysis}

Solid-state ${ }^{13} \mathrm{C}$-NMR spectra of samples (ca. $0.3 \mathrm{~g}$ ) of the MAKS were acquired using a Bruker AVANCE III $400 \mathrm{MHz}$ spectrometer (Bruker BioSpin $\mathrm{GmbH}$, Rheinstetten, Germany), operating at a frequency of $100.369 \mathrm{MHz}$, equipped with a zirconium oxide rotor (external diameter, $7 \mathrm{~mm}$, spun at $5 \mathrm{kHz}$ in a magic-angle spinning, MAS, probe). The crosspolarization contact time, pulse delay, and pulse number were $2.0 \mathrm{~ms}, 1 \mathrm{~s}$, and 10000 , respectively ${ }^{32}$.

\section{Animals}

Male SD rats (10 weeks old), specific pathogen free, provided by the Experimental Animal Centre of Xi 'An Jiaotong University College of medicine, China, were employed for all experiments. Animals were housed under controlled conditions $\left(23-27^{\circ} \mathrm{C}\right.$, relative humidity $50-60 \%, 12 \mathrm{~h}$ light $/ 12 \mathrm{~h}$ dark cycles) and allowed free access to food and water. All animals used in this experiment were cared for according to the ethical regulations for animal research of the Experimental Animal Centre of Xi'An Jiaotong University College of medicine.

\section{Experimental procedure}

Rats were divided into seven groups, including a control group (not receiving any treatment), a negative control group (receiving MAKS alone), a model group (receiving hydrazine alone), a positive control group (receiving both hydrazine and bifendate), and three experimental groups (receiving both hydrazine and MAKS). Each group consisted of six rats.

The model group was subjected to hepatic injury by intraperitoneal (i.p.) administration of hydrazine hydrate dissolved in sterile water with $\mathrm{pH}$ adjusted to 7.4 ( $65 \mathrm{mg} / \mathrm{kg}$ of free base). The positive controls also received gavage administration of bifendate $(10 \mathrm{mg} / \mathrm{kg})$ dissolved in sterile water at $\mathrm{pH}$ 7.4. The three experimental groups were allocated to gavage administration of MAKS at a low, medium, or high dose $(10,20$, and $30 \mathrm{mg} / \mathrm{kg}$, respectively) $30 \mathrm{~min}$ prior to the same hydrazine treatment. 
All animals were sacrificed by chloral hydrate anaesthesia $24 \mathrm{~h}$ after hydrazine exposure, after calculating specific weight loss, $a=(b-c) / b$, where $b$ is rat weight before MAKS or bifendate administration and $c$ is rat weight before chloral hydrate anaesthesia.

\section{Blood and tissue collection}

Femoral artery blood was collected for further analysis immediately after weighing the rats. It was allowed to clot and then the serum was separated by centrifugation at $1500 \mathrm{~g}$ for $10 \mathrm{~min}$ at $4^{\circ} \mathrm{C}$ and stored at $-80^{\circ} \mathrm{C}$ until determination of its ALT and AST activities (described below).

In addition, the liver of each rat was weighed and the liver index was calculated by dividing its weight by the weight of the rat before chloral hydrate anaesthesia. The liver was then divided into two roughly equal parts. One part was immediately fixed in freshly prepared $10 \%$ neutral buffered formalin at $4{ }^{\circ} \mathrm{C}$ for histopathological study. The other part was rinsed with saline solution, stored at $-80^{\circ} \mathrm{C}$, and subsequently homogenized in nine volumes of saline. The homogenate was centrifuged at $1500 \mathrm{~g}$ for $10 \mathrm{~min}$ and the supernatant was used as a total homogenized liver sample.

\section{Liver function assessment}

ALT and AST activities were determined using commercial assay kits supplied by Nanjing Jiancheng Bioengineering Institute, Nanjing, China. Biochemical evaluation of MAKS hepatoprotective activity SOD, GSH-Px and CAT activities, GSH contents, and MDA concentrations were also determined using commercial kits supplied by Nanjing Jiancheng Bioengineering Institute, Nanjing, China.

\section{Histological investigation}

For histopathological study, the part of each liver that had been fixed in formalin was embedded in paraffin wax. Sections of each liver $(5 \mu \mathrm{m}$ thick) were cut and stained with haematoxylin and eosin (H \& E). Inflammatory histological responses around the central vein (infiltration of inflammatory cells, vacuolar degeneration, and necrosis) were then observed under a microscope at $10 \times$ magnification.

\section{Statistical analysis}

All data are presented as means \pm SD. The effects of the treatments on the measured variables were evaluated by one-way ANOVA followed by Duncan's multiple range test. Statistical significance in the following text refers to $p<0.05$ unless otherwise stated.

\section{RESULTS}

\section{Elemental analysis}

Elemental amounts of C, H, N, and S in the MAKS samples were calculated. S:N ratios of eumelanin and pheomelanin are reportedly 0.01 and 0.46 , respectively ${ }^{34}$. We found that the $\mathrm{S}: \mathrm{N}$ ratio of MAKS was less than 0.01 , similar to that of the synthetic eumelanin, indicating that it consisted mostly of eumelanin. However, it had a lower $\mathrm{N}$ content and higher $\mathrm{C}: \mathrm{N}$ ratio (C 58.31, $\mathrm{N}$ 3.06) than the synthetic melanin (C 46.36, N 6.28), indicating that it contained more carboxylate and aliphatic groups. The elemental analysis suggests that it is structurally similar to melanin obtained from Taihe Black-bone silky fowl muscles ${ }^{1}$.

\section{UV-Vis absorption spectra}

MAKS and synthetic melanin exhibited very poor solubility in acidic aqueous solution and most organic solvents. Thus to acquire UV-Vis absorption spectra both melanins were dissolved in $0.2 \%$ ammonia, then the ammonia was removed using a rotary evaporator. As shown in Fig. 2, both MAKS and the synthetic melanin showed an absorption maximum at about $200 \mathrm{~nm}$, but a progressive decrease in absorbance with increasing wavelength after $200 \mathrm{~nm}$, in accordance with most previous analyses of melanin ${ }^{1,32}$. The slopes of linear plots are often used to identify melanin. The $\log$ of optical density of a melanin solution when plotted against wavelength produces a linear curve with negative slopes from -0.0015 to $-0.0030^{2}$. The slopes of the log-absorption curves of MAKS and the synthetic melanin were -0.00204 and -0.00195 , respectively, values which are in accordance with

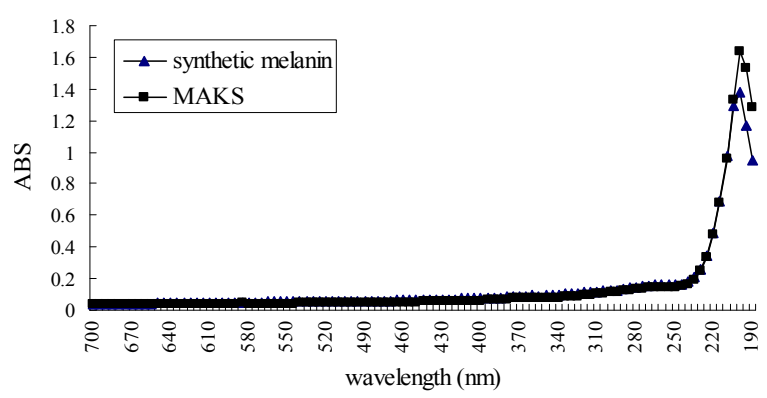

Fig. 2 UV-Vis absorption spectra (from 190-700 nm) of MAKS and synthetic melanin. 


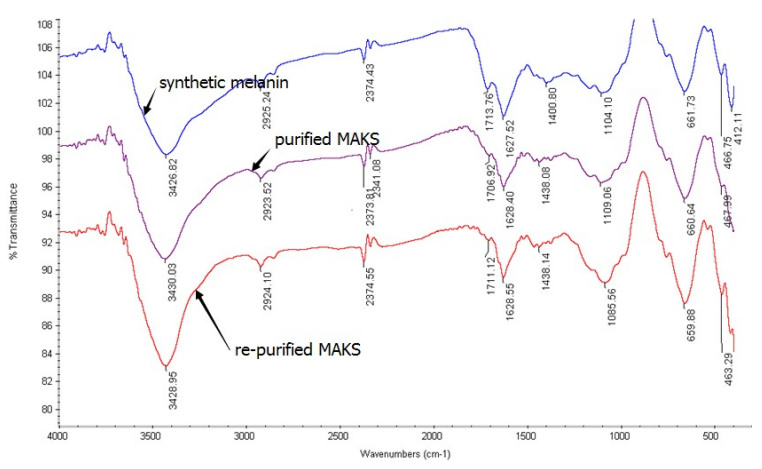

Fig. 3 IR spectra (from $4000-400 \mathrm{~cm}^{-1}$ ) of purified MAKS, re-purified MAKS, and the synthetic melanin.

the reported characteristics of melanins. In addition, MAKS exhibited no obvious absorption peak at $260 \mathrm{~nm}$ and $280 \mathrm{~nm}$, suggesting that MAKS has low nucleic acid and protein contents.

\section{Infrared spectra}

Infrared (IR) spectroscopy is an important nondestructive technique that can provide valuable information on functional groups present in small samples of a substance. The IR spectrum of the melanin extracted from apricot testae (Fig. 3) is similar to that of the synthetic melanin from 4000 to $400 \mathrm{~cm}^{-1}$, indicating that they have similar skeleton structures. Detailed analyses of the IR spectra of MAKS and previous studies suggest the following peak assignments: $-\mathrm{OH}, \mathrm{C}-\mathrm{H}$, or $\mathrm{N}-\mathrm{H}$ stretching $\left(3430 \mathrm{~cm}^{-1}\right), \mathrm{CH}_{2}-$ stretching $\left(2925 \mathrm{~cm}^{-1}\right), \mathrm{C}=\mathrm{O}$ stretching $\left(1710 \mathrm{~cm}^{-1}\right)$, aromatic groups stretching $\left(1630 \mathrm{~cm}^{-1}\right)$. These peaks, respectively, indicate the presence of $\mathrm{OH}$ (amino, phenol, and/or carboxylic groups), saturated alkyls, $\mathrm{C}=\mathrm{O}$ groups, and aromatic groups that may include amido, acylamino, conjugated quinones, and some carboxylic groups. These IR features correspond to the typical structure of melanin.

\section{MAKS molecular mass}

Gel chromatography resulted in the separation of two distinct MAKS fractions, as found for melanin derived from Chinese black tea ${ }^{23,33}$. However, their apparent molecular masses (derived from comparison of their elution volumes with those of the calibration standards) were much lighter $(2.4 \pm 0.12$ and $0.56 \pm 0.1 \mathrm{kDa}$ ) than those of the Chinese black tea melanins, 8 and $14.8 \mathrm{kDa}^{33}$. This may explain the darkness of Chinese black tea and lightness of apricot kernel skins.

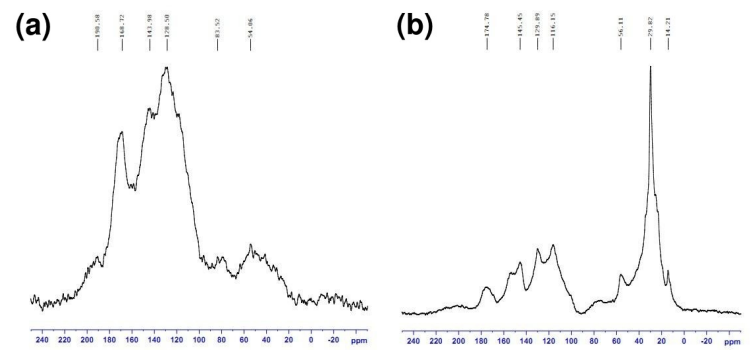

Fig. 4 Solid ${ }^{13}$ C-NMR spectra of (a) the synthetic melanin and (b) MAKS.

\section{${ }^{13} \mathrm{C}$-NMR spectra}

Solid-state NMR spectroscopy is a nondestructive technique that can provide complementary information to FT-IR spectroscopy in investigations of melanin chemical and structural properties. To assess distributions of the carbon species in the samples their NMR spectra were divided into the following four regions: (a) 0-51 ppm, representing saturated alkyl carbons; (b) 51-113 ppm, representing alkyl carbons substituted by heteroatoms such as $\mathrm{C}-\mathrm{O}$ in carbohydrates, $\mathrm{C}-\mathrm{N}$ in amino acids, and alkyl C-O; (c) 113-163 ppm, resulting from resonance of aromatic carbons, including resonance of phenolic groups in the 138-163 ppm subregion; and (d) 163-230 ppm, representing carbonyl carbons including carboxyl, amide, ester, and aldehyde carbons (particularly carboxyl carbons in the 163188 ppm subregion). The ${ }^{13} \mathrm{C}$-NMR spectra of both MAKS fractions showed broad resonance (Fig. 4), indicative of numerous overlapping signals and (hence) high heterogeneity. The spectra from both synthetic melanin and MAKS revealed the presence of alkyl, aromatic, phenolic, and carbonyl carbons, in accordance with their IR spectra. However, MAKS appears to have higher aliphatic carbon contents (manifested in strong signals at $20-40 \mathrm{ppm}$ ) and lower aromatic carbon contents than the synthetic melanin, like melanins from chestnut shells ${ }^{32}$.

\section{Rat hepatic injury induced by hydrazine}

The effects of MAKS on hydrazine hydrate-induced liver injury were studied in terms of changes in the rat behaviour, weight, hepatosomatic index, and biochemical markers. The model animals became inactive 2-3 h after i.p. injection of hydrazine at a dose of $65 \mathrm{mg} / \mathrm{kg}$, even refusing water or food. In contrast, after treatment with MAKS at a dose of $20 \mathrm{mg} / \mathrm{kg}$ prior to hydrazine the rats were more active and moderately consumed food and water. In addition, after receiving hydrazine alone the 
Table 1 Effects of hydrazine, MAKS, ${ }^{\dagger}$ and bifendate on rat weight loss and liver index.

\begin{tabular}{lcc}
\hline Group of animals & Weight loss (\%) & Liver index (\%) \\
\hline Control & $0.62 \pm 0.01^{\#}$ & $4.25 \pm 0.003^{\#}$ \\
Negative control & $0.79 \pm 0.03^{\#}$ & $4.28 \pm 0.005^{\#}$ \\
Model group & $8.73 \pm 0.04^{*}$ & $5.19 \pm 0.005^{*}$ \\
Positive control & $8.71 \pm 0.01^{*}$ & $4.78 \pm 0.002$ \\
MAKS low-dose & $5.52 \pm 0.02^{*}$ & $4.73 \pm 0.003$ \\
MAKS medium-dose & $10.14 \pm 0.02^{*}$ & $4.80 \pm 0.003$ \\
MAKS high-dose & $9.31 \pm 0.03^{*}$ & $4.76 \pm 0.002$ \\
\hline
\end{tabular}

$\dagger$ MAKS was given to rats $30 \mathrm{~min}$ prior to hydrazine administration (65 mg/kg, i.p.).

$*$ Control = no additives; negative control $=10 \mathrm{mg} / \mathrm{kg}$ MAKS; positive control $=20 \mathrm{mg} / \mathrm{kg}$ bifendate + hydrazine; model group = hydrazine; MAKS low, medium, and high doses $=12,20$, and $30 \mathrm{mg} / \mathrm{kg}$ MAKS + hydrazine, respectively.

*, \# Statistically significant $(p<0.05)$ differences, versus controls and model rats, respectively.

rat weight significantly decreased and their hepatosomatic index significantly increased (Table 1), possibly due to liver damage. However, the weight and hepatosomatic index of rats pretreated with MAKS also significantly decreased, indicating that the gavage could have caused the animals to refuse water and food, explaining the weight loss. Nevertheless, the reduction in their hepatosomatic index was substantially lower than that of the hydrazinetreated rats, confirming that MAKS could ameliorate liver enlargement induced by hydrazine.

\section{ALT and AST activities in serum}

ALT and AST activities in serum were used as biochemical markers for early acute hepatic damage ${ }^{5,25,26}$. As shown in Table 2 , these activities were significantly higher in hydrazine-treated rats than in the controls. MAKS pretreatment significantly and dose-dependently reduced the ALT and AST activities. Thus the results confirm that MAKS may ameliorate hepatic injury induced by hydrazine.

\section{Effect of MAKS on SOD activities}

SOD plays a key role in elimination of reactive oxygen species (ROS) derived from the peroxidation of xenobiotics in hepatic tissues ${ }^{26,29}$. As shown in Fig. 5, SOD activity was significantly lower in liver homogenates from the model rats $(75.4 \pm 2.8 \mathrm{U} / \mathrm{mg}$ protein) than in homogenates from the controls $(81.2 \pm 4.0 \mathrm{U} / \mathrm{mg}$ protein), and intermediate in homogenates from rats pretreated
Table 2 Effects of hydrazine, MAKS, and bifendate on serum ALT and AST activities.

\begin{tabular}{llc}
\hline Group of animals & ALT (U/l) & AST (U/l) \\
\hline Control & $42.6 \pm 3.4^{\#}$ & $96.6 \pm 4.0^{\#}$ \\
Negative control & $43.3 \pm 6.7^{\#}$ & $99.9 \pm 3.2^{\#}$ \\
Model group & $69.7 \pm 4.4^{*}$ & $164 \pm 18^{*}$ \\
Positive control & $54.2 \pm 2.9^{* \#}$ & $105.1 \pm 4.9^{\#}$ \\
MAKS low-dose & $60.8 \pm 3.9^{* \#}$ & $142 \pm 21^{* \#}$ \\
MAKS medium-dose & $55.8 \pm 3.1^{* \#}$ & $130 \pm 19^{* \#}$ \\
MAKS high-dose & $52.9 \pm 4.0^{* \#}$ & $121 \pm 12^{* \#}$ \\
\hline
\end{tabular}

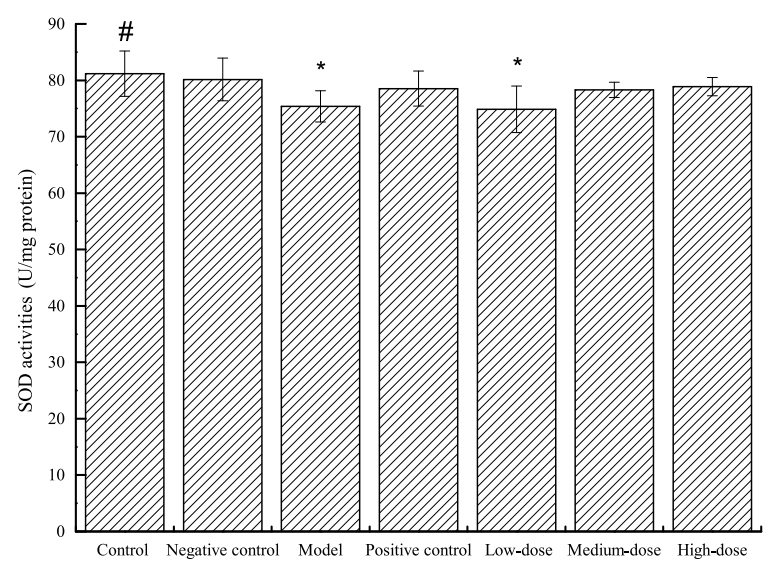

Fig. 5 Effects of hydrazine, MAKS, and bifendate on SOD activities in liver tissue.

with MAKS at medium or high doses $(78.3 \pm 1.4$ and $78.9 \pm 1.6 \mathrm{U} / \mathrm{mg}$ protein, respectively). There was no significant difference between the effects of administering hydrazine alone and in combination with MAKS, but there was a trend indicating that MAKS may enhance SOD activity and thus provide protection in responses to ROS.

\section{Effects of MAKS on GSH contents}

GSH is an intracellular reducing agent that plays major protective roles in the metabolism and transport of free radicals, peroxides, and other toxic compounds. Thus GSH deficiency in organisms can lead to tissue disorder and injury ${ }^{29}$. Significant reductions in GSH contents were detected in liver homogenates from hydrazine-treated rats $(0.09 \pm 0.04 \mathrm{~g} / \mathrm{mg}$ protein versus $5.45 \pm 0.74 \mathrm{~g} / \mathrm{mg}$ protein in homogenates from controls). At the $30 \mathrm{mg} / \mathrm{kg}$ dose, MAKS significantly ameliorated hydrazine-induced reduction of GSH in hepatic tissue, resulting in GSH contents of $1.34 \pm 0.12 \mathrm{~g} / \mathrm{mg}$ protein in the homogenates (Fig. 6). 


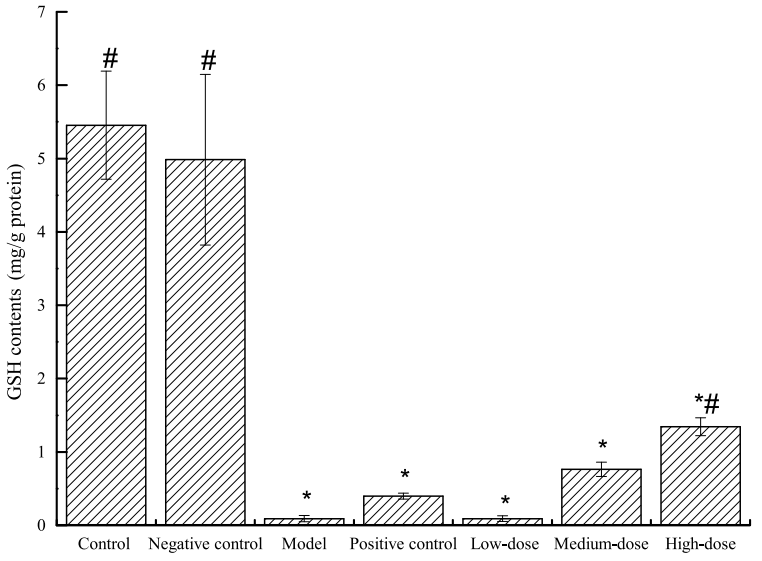

Fig. 6 Effects of hydrazine, MAKS, and bifendate on GSH contents in liver tissue.

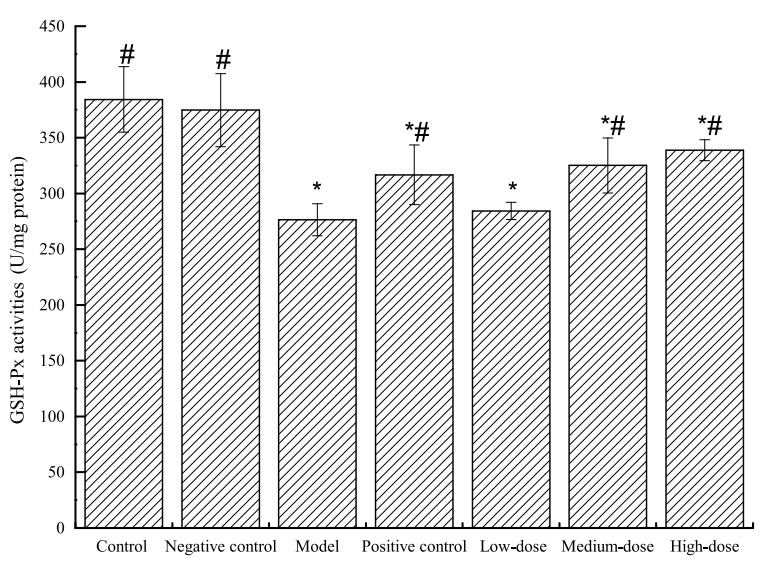

Fig. 7 Effects of hydrazine, MAKS, and bifendate on GSHPx activities in liver tissue.

\section{Effect of MAKS on GSH-Px activities}

GSH-Px activity was lower in liver homogenates from rats treated with hydrazine alone $(276 \pm 14 \mathrm{U} / \mathrm{mg}$ protein) than in controls (384 $\pm 29 \mathrm{U} / \mathrm{mg}$ protein), and intermediate in homogenates of rats pretreated with MAKS (Fig. 7). The activity was not significantly higher than in controls in homogenates from rats pretreated with the low dose, but significantly higher in homogenates from those pretreated with medium or high doses of $(325 \pm 25$ and $338.80 \pm 9.37 \mathrm{U} / \mathrm{mg}$ protein, respectively).

\section{Effect of MAKS on CAT activities}

CAT is a key component of the antioxidant defence system, inhibition of which results in enhanced sensitivity to free radicals and thus cellular damage $^{29}$. As shown in Fig. 8, CAT activity was con-

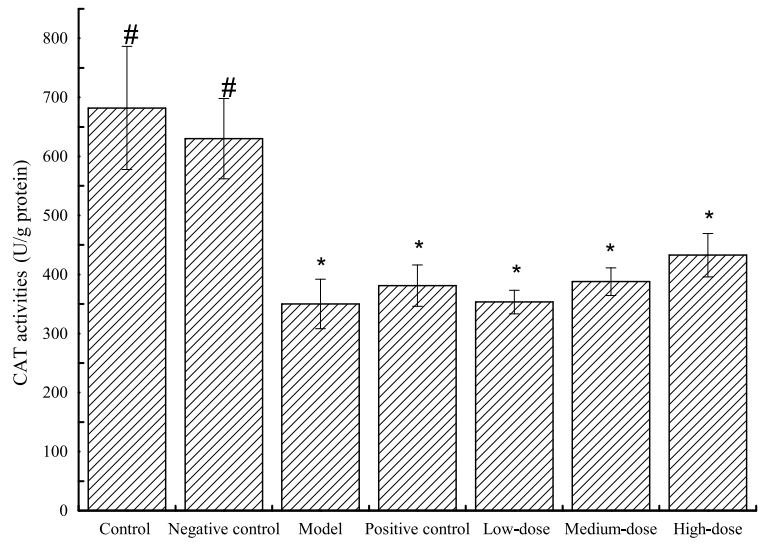

Fig. 8 Effects of hydrazine, MAKS, and bifendate on CAT activities in liver tissue.

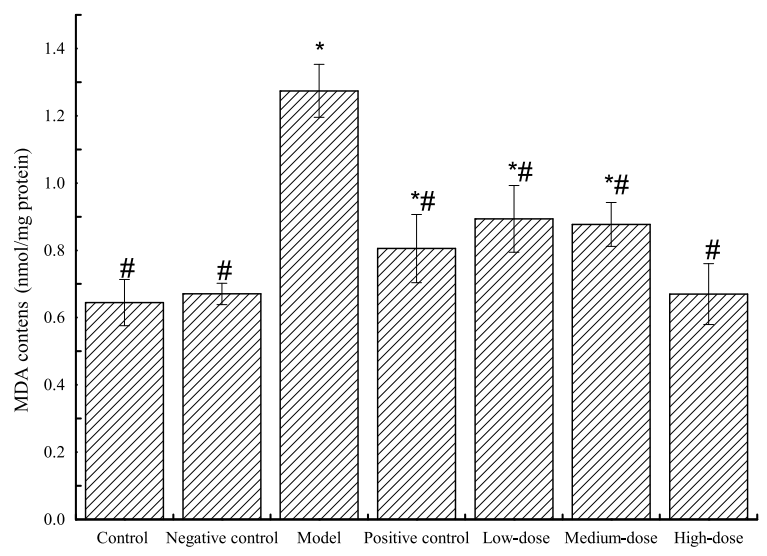

Fig. 9 Effects of hydrazine, MAKS, and bifendate on MDA concentration in liver tissue.

spicuously lower in liver homogenates from the rats treated with hydrazine alone ( $350 \pm 42 \mathrm{U} / \mathrm{g}$ protein) than in homogenates from controls $(682 \pm 104 \mathrm{U} / \mathrm{g}$ protein). The activities in homogenates from rats pretreated with MAKS were higher than in homogenates from model rats in a dose-dependent way. Thus CAT activity was increased by MAKS administration, suggesting that it can restore this activity to some degree.

\section{Effect of MAKS on MDA contents}

Hydrazine challenge caused a significant increase in lipid peroxidation (measured as increases in MDA contents) in hepatic tissue of the rats, as previously observed $^{5,28,29}$, to $1.27 \pm 0.08 \mathrm{nmol} / \mathrm{mg}$ protein, indicating hydrazine intoxication (Fig. 9). Pretreatment with MAKS dose-dependently reduced the MDA contents, and it completely restored control levels of MDA at $30 \mathrm{mg} / \mathrm{kg}$, indicating that it 

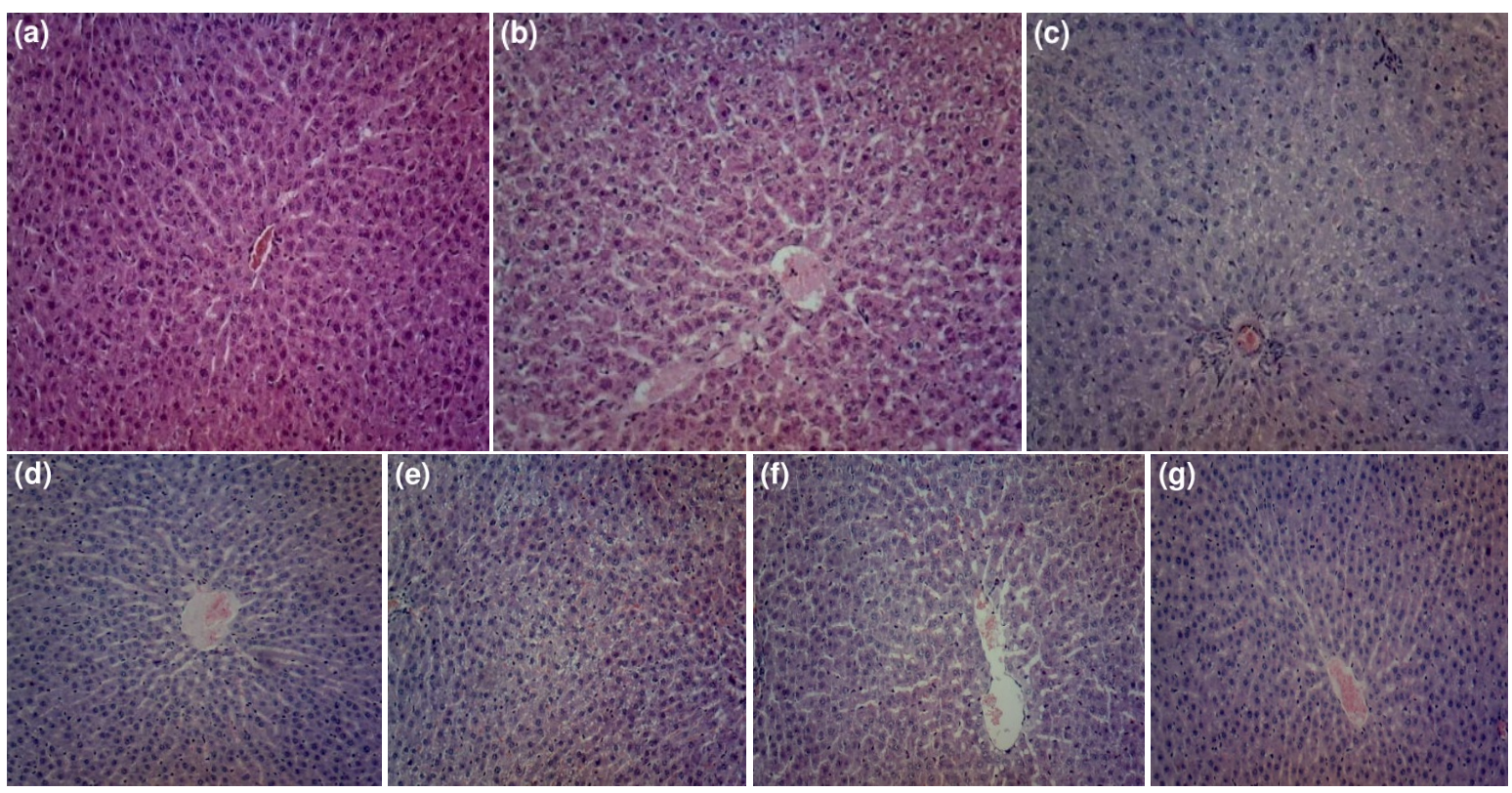

Fig. 10 Representative hepatic sections showing histopathological effects of the treatments: (a) control; (b) negative control; (c) model group; (d) positive control; (e) MAKS low dose; (f) MAKS medium dose; (g) MAKS high dose.

strongly inhibits hydrazine-induced lipid peroxidation.

\section{Effects of MAKS on liver histology}

Livers of the control and negative control rats had a normal lobular architecture with a central vein and radiations to hepatic cords, indicating that they were healthy and had no pathological perturbations (Fig. 10a,b). In contrast, severe changes were observed in livers from the hydrazine-treated rats, including multiple, extensive areas of portal inflammation and hepatocellular necrosis, randomly distributed throughout the parenchyma, and hepatocyte ballooning (Fig. 10c). Pretreatment with either MAKS or bifendate reduced the extent of necrotic zones, hepatocellular degeneration and destruction of the liver architecture, thus these agents apparently alleviated the liver damage (Fig. 10d-g). These histopathological observations corroborate the hepatoprotective potential of MAKS, confirming the results of the biochemical analyses.

\section{DISCUSSION}

Melanins have high potential to be used as natural colourants and antioxidants. They are large, extended, amorphous, irregular, heterogeneous, highly cross-linked, hydrophilic substances with complex, highly variable structures. They readily bind to other polymers, including proteins, polysaccharides, lipids, and other pigments and admix- tures. Due to their complexity, they are difficult to isolate and characterize using traditional chemical, histochemical, and physicochemical techniques $^{32,35}$. Thus the extraction and purification of melanins has usually involved the use of hot solvents, bases or acids, which can change the polymers or destroy their associations with other biological materials. A protocol involving alkaline extraction followed by purification by strong acid hydrolysis and washing with suitable solvents has been reported $^{32}$. However, the hydrolysis may change the chemical structure of melanins, particularly through extensive decarboxylation. Furthermore, little information on the structure of melanins is currently available. Nevertheless, UV-Vis, FT-IR, and solidstate nuclear magnetic resonance $\left({ }^{13} \mathrm{C}-\mathrm{NMR}\right)$ spectroscopy, molecular mass determinations, elemental analysis and other chemical analyses have provided valuable information on the structure of melanins from various sources ${ }^{1,16,32}$.

The presented study focused on the properties of melanin from apricot kernel skins (MAKS), which are currently waste products but have high melanin contents. For this purpose we applied a simple, inexpensive method to extract and fractionate MAKS, similar to procedures used to extract several other melanins and melanin-like substances ${ }^{14,23,32}$. The structural details obtained from subsequent analyses are described above in detail.

A number of drugs, chemicals, and viruses re- 
portedly cause severe liver necrosis, which can be difficult to treat $3,4,21,25,27,28$. Thus it is highly important to identify compounds that could ameliorate liver damage and hepatic failure. As melanin has ROS-scavenging activity ${ }^{1,5,36}$ and provides protection from UV irradiation ${ }^{37}$ we postulated that MAKS could protect against oxidant-mediated rat liver injury by attenuating oxidative stress and inhibiting inflammatory responses. Thus a main aim of the presented study was to test this hypothesis.

Hydrazine is an oxidant that is known to cause liver injury, including loss of functional integrity of cell membranes in liver tissue ${ }^{5,38}$. AST and ALT are released into the blood following liver damage, thus they have been used as biomarkers of hepatic damage $26,30,39$. Our results show that MAKS pretreatment markedly reduced serum AST and ALT activities in hydrazine-treated rats. These findings were corroborated by histopathological observations of affected livers. As shown both here and in a previous study ${ }^{25}$, hydrazine can cause various hepatic histological changes, including multiple, extensive areas of portal inflammation, hepatocellular necrosis randomly distributed throughout the parenchyma, and increases in inflammatory cell infiltration. Results presented here show that MAKS can attenuate these histological changes and thus significantly suppresses hydrazine-induced liver injury. The protective effects included inhibition of hydrazine-induced increases in serum ALT and AST activities, and MDA contents in the liver, together with inhibition of reductions in SOD, CAT, and GSHPx activities as well as GSH contents in rat liver tissues. Furthermore, administration of MAKS alone in the same time period did not affect the hepatic functional markers, indicating that it has low toxicity.

Based on the presented results we conclude that MAKS consists mostly of eumelanin and has very similar physiochemical characteristics to those of typical eumelanin. MAKS exhibited hepatoprotective effects on hydrazine-induced liver injury in rat, probably mediated by inhibition of increases in ALT and AST activities in serum and attenuation of oxidative stress in liver tissues, mediated by amelioration of increases in lipid peroxidation and reductions in GSH contents as well as SOD, CAT, and GSH-Px activities. Thus MAKS appears to be a potential therapeutic agent that can be safely administered at affective concentrations. Since MAKS has protective effects against liver injury, further investigations may lead to the development of new health products with attractive properties. Further research is planned to study the relationships between levels of oxidative DNA damage induced by hydrazine, tumour formation and MAKS administration in long-term feeding experiments.

Acknowledgements: This study was supported by a grant from the research and development of deep processing technology of kernel-apricot, PR China (Special Research Programme for Public-welfare Forestry of the Chinese State Forestry Administration No. 200904020).

\section{REFERENCES}

1. Chen SR, Jiang B, Zheng JX, Xu GY, Li JY, Yang $N$ (2008) Isolation and characterization of natural melanin derived from silky fowl (Gallus gallus domesticus Brisson). Food Chem 111, 745-9.

2. Ellis DH, Griffiths DA (1974) The location and analysis of melanins in the cell walls of some soil fungi. Can J Microbiol 20, 1379-86.

3. Manning JT, Bundred PE, Henzi P (2003) Melanin and HIV in sub-Saharan Africa. $J$ Theor Biol 223, 131-3.

4. Raimondi V, Agati G, Cecchi G, Gomoiu I, Lognoli D, Palombi L (2009) In vivo real-time recording of UV-induced changes in the autofluorescence of a melanin-containing fungus using a microspectrofluorimeter and a low-cost webcam. Opt Express $17,22735-46$.

5. Hung YC, Sava VM, Blagodarsky VA, Hong MY, Huang GS (2003) Protection of tea melanin on hydrazine-induced liver injury. Life Sci 72, 1061-71.

6. Herrling T, Jung K, Fuchs J (2008) The role of melanin as protector against free radicals in skin and its role as free radical indicator in hair. Spectrochim Acta A 69, 1429-35.

7. Kumar CG, Mongolla P, Pombala S, Kamle A, Joseph J (2011) Physicochemical characterization and antioxidant activity of melanin from a novel strain of Aspergillus bridgeri ICTF-201. Lett Appl Microbiol 53, 350-8.

8. Bafteh PR, Siegesmund M, Hanneken S, Neumann NJ (2012) Protective effects of $\beta$-carotene and melanin against protoporphyrine IX-induced phototoxicity in the photo hen's egg test. Photodermatol Photoimmunol Photomed 28, 12-6.

9. Ligonzo T, Ambrico M, Augelli V, Perna G, Schiavulli L, Tamma MA, Biagi PF, Minafra A, Capozzi V (2009) Electrical and optical properties of natural and synthetic melanin biopolymer. J Non Cryst Solid 355, 1221-6.

10. Yu SB, Geng J, Zhou P, Feng AR, Chen XD, Hu JM (2007) Analysis of plasmid DNA damage induced by melanin with capillary electrophoresis. $J$ Pharmaceut Biomed Anal 43, 816-21.

11. Schweitzer AD, Howell RC, Jiang Z, Bryan RA, Gerfen G, Chen CC, Mah D, Cahill S, et al (2009) Physico- 
chemical evaluation of rationally designed melanins as novel nature-inspired radioprotectors. PLOS ONE 4, e7229.

12. Mayer AM (2006) Polyphenol oxidases in plants and fungi: Going places? A review. Phytochemistry 67, 2318-31.

13. Pierce JA, Rast DM (1995) A comparison of native and synthetic mushroom melanins by fouriertransform infrared spectroscopy. Phytochemistry 39, 49-55.

14. Novellino L, Napolitano A, Prota G (2000) Isolation and characterization of mammalian eumelanins from hair and irides. Biochim Biophys Acta Gen Subj 1475, 295-306.

15. Latocha M, Chodurek E, Kurkiewicz S, Swiatkowska L, Wilczok T (2000) Pyrolytic GC-MS analysis of melanin from black, gray and yellow strains of Drosophila melanogaster. J Anal Appl Pyrol 56, 89-98.

16. Stevens LH, Davelaar E (1996) Isolation and characterization of blackspot pigments from potato tubers. Phytochemistry 42, 941-7.

17. Hanamura T, Hagiwara T, Kawagishi H (2005) Structural and functional characterization of polyphenols isolated from Acerola (Malpighia emarginata DC.) fruit. Biosci Biotechnol Biochem 69, 280-6.

18. Harki E, Talou T, Dargent R (1997) Purification, characterisation and analysis of melanin extracted from Tuber melanosporum Vitt. Food Chem 58, 69-73.

19. Riesz J, Gilmore J, Meredith P (2006) Quantitative scattering of melanin solutions. Biophys $J \mathbf{9 0}$, 4137-44.

20. Swan G A (1974) Structure, chemistry and biosynthesis of the melanins. In: Hertz W, Grisenbach H, Kirby GW (eds) Fortschritte der Chemie Organische Naturstoffe vol 31, Springer Verlag, Wien, pp 521-82.

21. Tu YG, Sun YZ, Tian YG, Xie MY, Chen J (2009) Physicochemical characterisation and antioxidant activity of melanin from the muscles of Taihe Blackbone silky fowl (Gallus gallus domesticus Brisson). Food Chem 114, 1345-50.

22. Zou Y, Hu WZ, Ma K, Tian MX (2015) Physicochemical properties and antioxidant activities of melanin and fractions from Auricularia auricula fruiting bodies. Food Sci Biotechnol 24, 15-21.

23. Hung YC, Sava VM, Makan SY, Chen THJ, Hong MY, Huang GS (2002) Antioxidant activity of melanins derived from tea-comparison between different oxidative states. Food Chem 78, 233-40.

24. Zhebentyayeva T, Ledbetter C, Burgos L, Llácer G (2012) Apricot. In: Badenes ML, Byrne DH (eds) Fruit Breeding, Handbook of Plant Breeding vol 8, Springer US, pp 415-58.

25. Shen X, Tang Y, Yang R, Fang T, Duan J (2009) The protective effect of Zizyphus jujube fruit on carbon tetrachloride-induced hepatic injury in mice by antioxidative activities. $J$ Ethnopharmacol 122, 555-60.

26. Cho BO, Ryu HW, So Y, Jin CH, Baek JY, Park KH,
Byun EH, Jeong IY (2013) Hepatoprotective effect of 2,3-dehydrosilybin on carbon tetrachloride-induced liver injury in rats. Food Chem 138, 107-15.

27. Tahir M, Rehman MU, Lateef A, Khan R, Khan AQ, Qamar W, Ali F,Hamiza O, Sultana S (2013) Diosmin protects against ethanol-induced hepatic injury via alleviation of inflammation and regulation of TNF- $\alpha$ and NF- $\kappa \mathrm{B}$ activation. Alcohol 47, 131-9.

28. Yamazaki M, Miyake M, Sato H, Masutomi N, Tsutsui N, Adam KP, Alexander DC, Lawton KA, et al (2013) Perturbation of bile acid homeostasis is an early pathogenesis event of drug induced liver injury in rats. Toxicol Appl Pharmacol 268, 79-89.

29. Hung YC, Sava VM, Makan SY, Hong MY, Huang GS (2004) Preventive effect of Thea sinensis melanin against acetaminophen-induced hepatic injury in mice. J Agr Food Chem 52, 5284-9.

30. Iwasaki Y, Tagaya N, Hattori Y, Yamaguchi K, Kubota K (2002) Protective effect of ischemic preconditioning against intermittent warm-ischemia-induced liver injury. J Surg Res 107, 82-92.

31. Sava VM, Hung YC, Golkin BN, Hong MY, Huang GS (2002) Protective activity of melanin-like pigment derived from tea on Drosophila melanogaster against the toxic effects of benzidine. Food Res Int $\mathbf{3 5}$, 619-26.

32. Yao ZY, Qi JH, Wang LH (2012) Isolation, fractionation and characterization of melanin-like pigments from chestnut (Castanea mollissima) shells. J Food Sci 77, C671-6.

33. Sava VM, Galkin BN, Hong MY, Yang PC, Huang GS (2001) A novel melanin-like pigment derived from black tea leaves with immuno-stimulating activity. Food Res Int 34, 337-43.

34. Ito S, Fujita K (1985) Microanalysis of eumelanin and pheomelanin in hair and melanomas by chemical degradation and liquid chromatography. Anal Biochem 144, 527-36.

35. Enochs WS, Nilges MJ, Swartz HM (1993) A standardized test for the identification and characterization of melanins using electron-paramagneticresonance (EPR) spectroscopy. Pigment Cell Res 6, 91-9.

36. Yao ZY, Li KY, Zhao Zh, Ma XH (2007) Antioxidant properties of melanin from testae of Armeniaca vulgaris var. ansu. Sci Silvae Sin 43(10), 59-63.

37. Brenner M, Hearing VJ (2008) The protective role of melanin against UV damage in human skin. Photochem Photobiol 84, 539-49.

38. Daglar G, Karaca T,Yuksek YN, Gozalan U, Akbiyik F, Sokmensuer C, Gurel B, Kama NA (2009) Effect of montelukast and MK-886 on hepatic ischemiareperfusion injury in rats. $J$ Surg Res 153, 31-8.

39. Li JX, Li HJ, Zhao Zh, Xia QM, Zhu HL (2012) Optimization of extraction process for melanin pigment from apricot kernel skin. Food Sci 33(8), 11-4. 